\title{
La diversité de points de vue des auteurs musulmans sur la dissimulation du visage
}

Moussa Abou Ramadan

\section{OpenEdition}

Édition électronique

URL : http://journals.openedition.org/rdr/940

DOI : $10.4000 /$ rdr.940

ISSN : 2534-7462

Éditeur

Presses universitaires de Strasbourg

\section{Édition imprimée}

Date de publication : 8 novembre 2016

Pagination : 107-119

ISBN : 978-2-86820-959-7

ISSN : 2493-8637

Référence électronique

Moussa Abou Ramadan, «La diversité de points de vue des auteurs musulmans sur la dissimulation du visage », Revue du droit des religions [En ligne], 2 | 2016, mis en ligne le 11 février 2020, consulté le 19 novembre 2020. URL : http://journals.openedition.org/rdr/940 ; DOI : https://doi.org/10.4000/rdr. 940

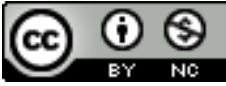

La revue du droit des religions est mise à disposition selon les termes de la Creative Commons Attribution - Pas d'Utilisation Commerciale 4.0 International - CC BY-NC 4.0. 


\section{LA DIVERSITÉ DE POINTS DE VUE DES AUTEURS MUSULMANS SUR LA DISSIMULATION DU VISAGE}

\section{Moussa ABOU RAMADAN}

Université de Strasbourg / CNRS, Droit, Religion, Entreprise et Société (DRES)

\section{RÉSUMÉ}

Nous examinerons successivement dans cet article la position des juristes de trois écoles juridiques sunnites, les opinions des auteurs salafistes contemporains et la position du cheikh al-Qaradawi, avant d'exposer le point de vue des coranistes, particulièrement celle de Jamal al-Bana. En étudiant les opinions des auteurs musulmans sur la question de la dissimulation du visage, il ressort, tant chez les auteurs classiques que chez les auteurs contemporains, une absence d'unanimité, y compris au sein de chaque courant.

\section{ABSTRACT}

In this article we examine the positions of the jurists of three Sunni legal schools, the opinions of contemporary Salafi authors and the position of Sheikh Yusuf al-Qaradawi before turning to the views held by Qur'anists, particularly those of Gamal al-Banna. Studying the opinions of the Muslim authors on the issue of the covering of the face allows discerning that there is apparently a lack of unanimity among both classical and contemporary authors, even within each current. 
$\mathrm{D}$ ans son livre classique sur la sexualité en islam, Abdel Wahab Bouhdiba ${ }^{1}$ traite de la question du hijab (voile) dans le chapitre qui étudie la dichotomie entre les deux sexes masculin et féminin. Les symboles établissant une frontière entre l'homme et la femme sont le voile pour celle-ci et la barbe pour celui-là. Une autre signification s'est ajoutée à la première - séparation entre homme et femme -, il s'agit de l'affirmation de l'identité musulmane en plus de celle de la féminité. Le voile intégral (niqab) comporte lui un troisième sens qui vise à distinguer non pas seulement l'homme de la femme, le musulman du non-musulman, mais aussi le « vrai » musulman du «faux» musulman ${ }^{2}$, le vrai musulman étant celui qui reconnaît le voile intégral comme obligatoire.

Nous examinerons successivement la position des juristes de trois écoles juridiques sunnites, les opinions des auteurs salafistes contemporains et la position du cheikh al-Qaradawi avant d'exposer pour finir le point de vue des coranistes, particulièrement celle de Jamal al-Bana. En étudiant les opinions des auteurs musulmans sur la question de la dissimulation du visage, il ressort, tant chez les auteurs classiques que chez les auteurs contemporains, une absence d'unanimité, y compris au sein de chaque courant. Les opinions sur le voile intégral en droit musulman classique (1) se divisent entre celles qui tolèrent la non-couverture du visage et celles qui prescrivent la dissimulation de ce dernier. À l'époque contemporaine (2), la position salafiste prescrit la dissimulation du visage et considère ceux qui prônent le contraire comme des infidèles. Les fuqaha'3 du Moyen-Orient, dont al-Qaradawi, autorisent la divulgation du visage ; d'autres penseurs musulmans, comme Jamal al-Bana, ne considèrent même pas le voile non intégral comme obligatoire.

\section{LA DISSIMULATION DU VISAGE EN DROIT MUSULMAN CLASSIQUE}

Les juristes musulmans n'ont pas consacré de chapitre ou de livre sur la dissimulation du corps à l'exception de l'ouvrage du XIII ${ }^{\mathrm{e}}$ siècle de al-Qatan al-Fasi (mort en 1231) dans lequel l'auteur examine le statut du regard ${ }^{4}$. Chez la plupart des fuqaha' cette question est éparpillée dans différents cha-

1. Boundiba A., La sexualité en islam, Paris, PUF, 1975.

2. Benkheira M. H., « Le visage de la femme. Entre la sharîa et la coutume », Anthropologie et Sociétés, vol. 20, n 2, 1996, p. 15-36.

3. Juristes musulmans qui sont experts en doctrine juridique musulmane (fiqh).

4. Al-Nazar fi ahkam al-nazar bi-hasat al-nazar, Tanata, Dar al-sahaba lil turath, 1994. Sur ce livre, V. Chaumont É., « La notion de 'awra selon Abu-l-Hassan 'Ali b. Muhammad 
pitres de traités de fiqh (doctrine juridique musulmane) consacrés à la prière (salat), la toilette funéraire ou les fiançailles; pour l'école hanafite ${ }^{5}$, elle est traitée dans le chapitre sur l'équité (istihsan). Le terme utilisé par al-Qatan al-Fasi pour évoquer la question du regard est 'awra provenant du mot a'war « borgne » et traduit par Boudhiba ${ }^{6}$ comme «partie intime ». Éric Chaumont le traduit comme une partie du corps ne pouvant pas être révélée et devant être dissimulée au regard ${ }^{7}$.

Cette question de l'obligation ou non de la dissimulation du visage dépend de plusieurs facteurs et ce n'est pas une obligation absolue, même pour celui qui défend la thèse de la dissimulation. Plusieurs éléments interviennent : le genre (le regard d'une femme ou d'un homme); le statut social (libre ou esclave); les relations familiales (mari, fiancé, personne avec un lien de parenté à un degré prohibant le mariage, autres personnes); la situation juridique dans laquelle on se trouve (prière, ihram ${ }^{8}$, toilette funéraire, traitement médical, témoignage devant un tribunal); la force ou la faiblesse du désir sexuel de l'homme; l'âge (majeur(e), mineur(e), vielle femme qui n'est pas désirée) ; la religion des personnes ${ }^{9}$. Tous ces facteurs alimentent la casuistique juridique en la matière.

b. al-Qattan al-Fasi (m. 628/1231) », Revue des mondes musulmans et de la Méditerranée, $n^{\circ} 113-114,2006$, p. 109-123.

5. La religion musulmane est divisée en trois tendances principales : les sunnites, les chiites et les kharijites. Cet article examine uniquement les positions des sunnites qui constituent environ $90 \%$ des musulmans. En matière juridique, les sunnites connaissent quatre écoles juridiques qui ont bâti leurs propres méthodologies, philosophies du droit et contenus des droits : l'école hanafite, malékite, chaféite et hanbalite.

6. Boundiba A., op. cit., p. 52.

7. Chaumont É., art. cit., p. 113.

8. Ihram est traduit en français comme sacralisation, mais ce mot désigne plus précisément la période de pèlerinage à la Mecque d'un musulman ou d'une musulmane. Pendant cette période, certaines interdictions incombent aux croyants, par exemple l'interdiction à la femme de couvrir son visage.

9. Al-Ghazali (m. 505/1111), Al-Wasit fi al-madhhab, Le Caire/Dar al Salam, 1417, vol. 2, p. 175-176, vol. 5, p. 28-37. - Al 'IMrani (m. 558/1163), Al-Bayan fi madhhab al-imam al-Shafi'I, Jadda, Dar al-minhaj, 2000, vol. 2, p. 117-121 et vol. 9, p. 125-133. - ALMAWAQ (m. 897/1492), Al-Tal wa al-iqlil li mukhtassar Khalil, Dar al-kutub al-'ilmiyya, 1994, vol. 2, p. 181. - Al-HаттAв (m. 954/1547), Mawahib al-jalil fi sharh mukhtassar kalil, Dar al-fikr, 1992, vol. 1, p. 499-501. - Al-KaSANi (m. 587/1191), Bada'i' al-sana'i' fi tartib al-shara'i', Dar al-kutub al 'ilmiyya, 1986, vol. 5, p. 118-123. 


\subsection{LE STATUT JURIDIQUE DU REGARD ET CE QUI PEUT ÊTRE OU PAS RÉVÉLÉ}

Pour les hanafites, al-Sarakhsi (m. 490/1097) ${ }^{10}$, se basant sur al - Kirkhi (m. 340/952), classe le statut juridique du regard d'une personne sur une autre en quatre catégories s'agissant des personnes libres : le regard de l'homme sur l'homme, le regard de la femme sur la femme, le regard de la femme sur l'homme et le regard de l'homme sur la femme ${ }^{11}$.

Concernant la première catégorie (le regard de l'homme sur l'homme), le 'awra se situe du nombril jusqu'aux genoux. Pour al-Sarakhsi, le nombril n'est pas 'awra si l'on tient compte que Abu Hurayra a embrassé le nombril du Prophète, qu'Ibn Omar laissait apparaître son nombril et que les personnes qui fréquentent le hammam laissent entrevoir leur nombril. La deuxième catégorie, à savoir le regard de la femme sur la femme, contient les mêmes règles que le regard de l'homme sur l'homme. Al-Sarakhsi mentionne que la femme fait la toilette funéraire de la femme et donc qu'elle a un droit de regard sur elle. Certains considèrent que la femme ne peut voir le ventre ou le dos d'une autre femme, en se référant à un hadith du Prophète qui interdit le hammam aux femmes ${ }^{12}$. Al-Sarakhsi estime que ce hadith évoque non pas le hammam, mais le fait que les femmes doivent rester dans leur maison. Il ajoute que l'on trouve des hammams pour femmes dans tous les pays et que ces dernières ont plus besoin que les hommes de fréquenter le hammam pour leurs ornements (zina), les hommes pouvant se laver dans les rivières alors que les femmes ne le peuvent pas. Pour la troisième catégorie concernant le regard de la femme sur l'homme, al-Sarakhsi rapporte deux opinions : l'une indiquant que ce qui n'est pas 'awra peut être regardé, qu'il s'agisse d'un homme ou d'une femme, l'autre estimant que ce n'est pas exact, car la femme ne peut pas faire la toilette funéraire d'un homme. Or, si le regard était le même, la femme pourrait l'effectuer. Ici, al-Sarakhsi introduit un élément subjectif en disant que tout dépend du shahwa, c'est-à-dire du désir sexuel : si ce désir existe, le regard n'est pas possible.

Pour la quatrième catégorie, à savoir le regard de l'homme sur la femme, elle se divise en quatre sous-catégories : la première concerne le regard de l'homme sur son épouse et sur son esclave; la deuxième concerne le regard de l'homme sur les femmes avec lesquelles il y a un degré de prohibition du

10. Al-Sarakhsi, Al-Mabsut, Beyruth, Dar al-ma 'rifa, 1993, vol. 10, p. 145.

11. Ces catégories se trouvent aussi chez al-Qatan al-Fasi, Al-Nazar, op. cit.

12. Sur cette question du hammam, V. plus longuement: BenkHEIRA M. H., « Hammam, nudité et ordre moral dans l'islam médiéval », Revue de l'histoire des religions, 2007, p. 319-371 et 2008, p. $75-128$. 
mariage ; la troisième concerne l'esclave d'un autre maître ; la quatrième souscatégorie concerne le regard sur une femme libre étrangère (qui n'a pas de lien de prohibition du mariage). Dans le premier cas, il est légal pour l'homme de pouvoir porter le regard sur tout le corps de l'épouse ou de l'esclave, de la tête jusqu'aux pieds, avec ou sans shahwa (désir). Al-Sarakhsi se base sur un hadith du Prophète disant « Ne regarde pas les femmes sauf ton épouse et ton esclave ». Un autre argument repose sur le fait que la femme du Prophète 'Aysha, racontait que le Prophète et elle-même se lavaient ensemble. Malgré cela, al-Sarakhsi estime qu'il est préférable de ne pas regarder le 'awra, car ni 'Aysha ni le Prophète n'ont vu le 'awra de l'autre, le 'awra étant entendu ici comme les organes sexuels. Une opinion d'autres juristes musulmans dit que le regard sur le sexe de la femme rend aveugle, certains le réfutant et défendant même l'idée que le hadith sur lequel est basé cette opinion est un hadith forgé (inventé) ${ }^{13}$.

Dans le deuxième cas concernant le regard sur les femmes qu'on ne peut épouser à cause d'empêchements permanents comme la filiation, le statut marital et l'allaitement, l'homme peut regarder zina zahira et zina batina (sourate La lumière, verset 31), c'est-à-dire les atours apparents et les atours dissimulés. Ici, il ne s'agit pas des atours proprement dits, parures que l'on peut acheter au marché et que tout le monde peut voir, mais des parties du corps qui portent ces ornements : la tête qui porte la couronne, le cou et la poitrine qui portent le collier, les oreilles qui portent les boucles, la main qui porte les bagues...

La troisième sous-catégorie évoque le regard sur l'esclave d'un autre maître, et ce sont ici les mêmes règles qui s'appliquent que pour le regard des hommes sur des femmes avec lesquelles il y a un degré de prohibition $\mathrm{du}$ mariage. Les fuqaha' estiment que Dieu a ordonné aux femmes libres de porter le jilbab (vêtement couvrant) pour se distinguer justement des femmes esclaves.

La quatrième sous-catégorie concerne le regard sur les femmes étrangères, dont les hommes peuvent regarder les atours apparents et non les atours dissimulés. Pour 'Ali et Ibn 'Abbas, cousins du prophète, les atours apparents, c'est par exemple le khôl. Pour 'Aysha, le regard créerait la fitna (dissension,

13. Dasuqui (m. 1230/1815), Hashiyat al-Dasuqui 'ala al-sharh al-kabir, Le Caire, Dar ihya' al turath al 'arabiya, s.d., vol. 2 p. 215. - IBN HAzm (m. 456/1064), Al-Muhala, Le Caire, Al-tiba'a al-munira, 1933, vol. 10, p. 33. - Muhamad NASir Al-Din Al-Albani, Silsila alahadith al-da'ifa wa al-mawdu'a wa atharaha al-sayi' fi al-umma, Riyad, Maktaba al-ma'arif, 1992, vol. 1, p. 351. 
désordre) et, par peur de la fitna, il faut tout cacher. Si l'œil n'est pas caché, c'est par nécessité (darura), afin que la femme puisse voir le chemin en marchant. Les hanafites ont quant à eux choisi l'opinion d'Ibn 'Abbas et d'Ali qui permet de ne pas dissimuler le visage, en se basant sur plusieurs écrits : une femme s'est présentée au Prophète pour qu'il l'épouse et a montré son visage qui ne lui a pas plu; on rapporte qu'Omar a discuté avec une femme dont le visage était découvert... Tout cela est permis à condition qu'il n'y ait pas shahwa (désir). Si, en revanche il y a shahwa, l'homme ne devra pas porter le regard sur la femme, mais sans qu'il soit pour autant interdit pour elle de se dévoiler. On se base entre autres sur un hadith du Prophète selon lequel un homme lui disait avoir regardé une femme en la désirant et l'avoir suivie du regard jusqu'à ce que sa tête ait heurté un mur. Le Prophète lui a répondu que si Dieu veut faire du bien avec une personne, il la punit dans ce monde. Les fuqaha' en déduisent que le regard avec désir est interdit. D'autres juristes hanafites appuient aussi cette position qui considère qu'il n'est pas obligatoire pour une femme libre de se dissimuler le visage devant des hommes avec lesquels il n'y a pas de degré de prohibition du mariage ${ }^{14}$.

\subsection{DES POSITIONS DIVERGENTES QUANT À CE QUI PEUT ÊTRE VISIBLE}

Pour les chaféites, le 'awra de la femme libre correspond à tout son corps, sauf les mains (kafayha) et le visage. Al-Shirazi (m. 476/1083) ${ }^{15}$, explique cette position en citant également le verset 31 de la sourate La lumière et rapporte le commentaire d'Ibn 'Abbas sur les atours : ce qui reste visible, ce sont le visage et les mains. Un autre argument réside dans le fait que le Prophète a interdit aux femmes musulmanes de mettre des gants (kafazin) et un niqab pendant l'ihram ${ }^{16}$. Si le visage et les mains étaient 'awra, il n'y aurait pas cette interdiction de les couvrir quand elles sont en état d'ihram. Un troisième argument avancé énonce qu'il est nécessaire de découvrir le visage lors de la vente et de l'achat et de découvrir les mains pour donner et prendre.

14. Al-Sughdi (m. 461/1069), Al-Nutaf fi al-fatawi, Aman/Beyrouth, Dar al furqan/muasasat al risala, 1984, vol. 1, p. 60. - Al-KASANI, op. cit., p. 121. - Al-MARGHINANi (m. 593/1197), Al-Hidaya fi sharh bidaya al-mubtadi', Beyrouth, Dar ihya' al-turath al 'arabi, vol. 4, p. 368 : il justifie la non-dissimulation du visage par la nécessité de faire des transactions avec les hommes, en donnant et en prenant.

15. Al-Muhazib fi fiqh al-Shafi 'i, Dar al-kutub al-'ilmiya, vol. 1, p. 124. - V. aussi AL-RAFI 'I (m.623/1226), Fath al 'aziz bi sharh al wajiz (dar al-fikr), vol. 4, p. 87.

16. V. note 8 . 
Chez les malékites, Ibn Rush (m. 595/1199), connu sous le nom d'Averroès, rapporte les divergences de points de vue entre deux positions. La première position est celle de la majorité qui voit dans la définition de 'awra tout le corps de la femme, sauf son visage et ses mains. Abou Hanifa (m. 150/767) considère par ailleurs que les pieds (kadamayha) ne sont pas 'awra. Puis Ibn Rush mentionne une opinion minoritaire d'Abu Bakr Bin 'Abdil Rahman et Ibn Hanbal (m. 241/855), selon laquelle tout le corps de la femme est 'awra. Il essaie d'expliquer les raisons de leurs divergences sur ce point en citant là encore le verset 31 de la sourate La lumière : parle-t-on de parties précises du corps ou bien s'agit-il de ce qu'on ne peut pas dévoiler ? Ceux qui penchent pour cette dernière proposition considèrent que tout le corps est 'awra. D'autres juristes prennent en compte également le verset 59 de la sourate Les factions et s'appuient sur le fait que pendant le pèlerinage (hajj), la femme ne dissimule pas son visage. Plus tard, un autre juriste malékite, al-Mawaq (m. 897/1492) rapporte l'avis de Malik selon lequel une femme peut manger devant une autre personne. Ibn al-Qatan en déduit que la femme peut découvrir son visage et ses mains devant les hommes. Selon Ibn Muhriz le visage de la femme n'est pas 'awra. De même le fait que le Prophète n'a pas permis de porter à deux reprises le regard sur une femme signifie que la dissimulation du visage n'est pas obligatoire ${ }^{17}$. Ibn al-Hattab confirme que la femme n'est pas obligée de dissimuler son visage ${ }^{18}$, mais il rapporte également l'opinion du cadi 'Abdil Wahab (m. 422/1031) selon laquelle s'il y a un risque de désordre (fitna) à cause d'une femme, celle-ci est alors obligée de dissimuler son visage et ses mains.

\section{LA POSITION DES FUQAHA' ET PENSEURS MUSULMANS CONTEMPORAINS}

Nous évoquerons ici, sans être exhaustif, des positions représentatives des différents courants de pensée contemporains.

Le salafisme signifie littéralement le retour aux salaf (prédécesseurs), c'està-dire aux premières générations de l'islam, afin d'accéder à l'islam " pur », avant sa "dégénérescence » et sa « contamination ». Au XIX siècle, le courant

17. Al-Mawaq (m. 897/1492), Al-Tal wa al-iqlil li mukhtasar Khalil, Dar al-kutub al-'ilmiya, 1994, vol. 2, p. 181.

18. V. aussi Al-Khurashi (m. 1101/690), Sharh muktasar Khalil lil Khurashi, Beyrouth, Dar al fikkr lil tiba'a, vol. 1, p. 247. 
réformiste de l'islam relancé par Muhammad 'Abdu (m. 1323/1905) essaie de retourner directement aux sources pour présenter le «pur» islam, dans le but de l'adapter à son époque. La même idée de retour aux sources a été développée également par Muhammad Bin 'Abdil Wahab (m. 1206/1792), fondateur du mouvement wahhabite en Arabie saoudite. Chaque courant retourne aux fondements, mais chacun en y trouvant un islam différent. Aujourd'hui, on utilise le terme salafisme pour désigner l'islam conservateur, qu'il soit quiétiste ou jihadiste.

\subsection{IBN BAZ : IL FAUT DISSIMULER LE VISAGE}

Comme exemple du salafisme, nous analyserons la position d'Ibn Baz (m. 1999) qui fut président du Haut comité des oulémas en Arabie saoudite. Dans une épître ${ }^{19}$ portant sur les règles de non-dissimulation du visage et sur le foulard, il regrette le fait que les femmes musulmanes ne portent pas le voile, ce qui constitue selon lui un grand péché. Il considère en effet que le foulard est obligatoire, ainsi que la dissimulation du visage, sur la base des arguments suivants issus de la sourate Les factions, verset 32 : "Ô femmes du prophète! Vous n'êtes semblables à nulle autre femme. Si vous êtes pieuses, ne soyez pas (trop) humbles en vos dires, en sorte que vous convoite celui au cœur duquel est un mal! Tenez (plutôt) un langage reconnu (pertinent)!» et verset 33 : «Demeurez dans vos demeures! Ne vous produisez point en vos atours, à la manière de l'ancienne Gentillité (jâhiliyya)! Accomplissez la Prière! Donnez l'Aumône (zakât). Obéissez à Allah et à Son Apôtre! ». Il s'agit d'un ordre divin adressé aux épouses du Prophète de ne pas adoucir leurs paroles envers les hommes pour ne pas susciter leur désir sexuel, ainsi que de demeurer dans leurs maisons et de ne pas faire apparaître leur visage, cou, poitrine, mains, pieds, car cela constitue une corruption et une grande fitna qui peut mener à la fornication. Bien que ce verset soit adressé aux épouses du Prophète, sa portée est générale selon Ibn Baz, et cela pour deux raisons. Il précise tout d'abord que si ces paroles sont destinées aux femmes du Prophète qui sont connues pour leur pureté, leur croyance et leur droiture, elles sont a fortiori adressées également aux autres femmes. Ensuite, la fin du verset 33 mentionne l'accomplissement de la prière et le don de l'aumône, deux obligations qui incombent à toutes les femmes quelles qu'elles soient. Puis Ibn Baz cite une phrase du verset 53 de la sourate Les factions : "Quand vous leur demandez

19. IBN BAz, Risala tabhath fi masa’il al-hijab wa al-sufur. 
un objet, demandez-le derrière un voile. ${ }^{20}$ Ici Ibn Baz ne fait pas l'effort de préciser que ce verset est adressé aux épouses du Prophète et il le prend comme un verset général s'appliquant à toutes les femmes, car pour lui le foulard est pureté et la non-couverture est najasa (impureté). Il cite encore le verset 59 de la même sourate : "Ô Prophète! dis à tes épouses, à tes filles et aux femmes des Croyants de serrer sur elles leurs voiles! Cela sera le plus simple moyen qu'elles soient reconnues et qu'elles ne soient point offensées. Allah est absoluteur et miséricordieux. " Il interprète ici le voile comme dissimulant non seulement les cheveux, mais aussi le visage. Selon le verset 60 de la sourate La Lumière : "Nul grief aux femmes atteintes par la ménopause et n'espérant plus mariage si elles déposent leurs voiles, (sauf à) se montrer sans atours. S'abstenir est toutefois un bien pour elles. Allah est audient et omniscient. » Les vieilles femmes qui ne sont pas désirées pour le mariage ne sont pas obligées de couvrir leurs visages et leurs mains, sauf si elles montrent leurs atours et dans ce cas l'obligation de couvrir leur visage réapparaît. Aussi, Ibn Baz soutient ici un argument énonçant que si l'obligation de se voiler quand la femme montre ses atours réapparaît, elle incombe a fortiori aux jeunes femmes et aux belles femmes. Puis Ibn Baz cite deux autres versets de la sourate La lumière : verset 30 : « Dis aux Croyants qu'ils baissent leurs regards et soient chastes. Ce sera plus décent pour eux. Allah est bien informé de ce qu'ils font » et verset 31 :

« Dis aux Croyantes de baisser leurs regards, d'être chastes, de ne montrer de leurs atours que ce qui en paraît. Qu'elles rabattent leurs voiles sur leurs gorges! Qu'elles montrent seulement leurs atours à leurs époux, ou à leurs pères, ou aux pères de leurs époux, ou à leurs fils, ou aux fils de leurs époux, ou à leurs frères, ou aux fils de leurs frères, ou aux fils de leurs sœurs, ou à leurs femmes, ou à leurs esclaves, ou à leurs serviteurs mâles que n'habite pas le désir (charnel), ou aux garçons qui ne sont pas (encore) au fait de la conformation des femmes. Que (les Coyantes) ne frappent point (le sol) de leurs pieds pour montrer les atours qu'elles cachent! Revenez tous à Allah, ô Croyants! Peut-être serez-vous bienheureux.»

Concernant la première phrase de ce verset, Ibn Baz constate qu'il y a une divergence de point de vue sur l'interprétation de « ce qui en paraît ». Ibn

20. Nous avons modifié la traduction de Régis Blachère (Le Coran, Paris, Maisonneuve et Larose, 1966), car celle-ci se lit comme suit : "Quand vous demandez un objet aux (épouses du Prophète) demandez-le derrière un voile. » Le traducteur a ajouté le texte entre parenthèses qui ne s'impose pas quand le verset est cité en entier, puisqu'il est question des femmes du Prophète avant et après cette phrase citée par Ibn Baz. 
Mas'ud, compagnon du Prophète, aurait interprété cela comme étant les vêtements que l'on n'est pas obligé de dissimuler et Ibn 'Abass comme désignant les mains et le visage. Mais Ibn Baz soutient que cette dernière interprétation n'est plus valable, car elle est antérieure à la révélation des versets sur le hijab dans la sourate Les factions. Il fait référence ici à la théorie de l'abrogation (naskh) selon laquelle s'il y a une contradiction entre deux versets sans moyen de les concilier, il faut appliquer le verset révélé postérieurement ${ }^{21}$. Puis il rapporte des hadiths qui permettent la non-dissimulation du visage, notamment celui dans lequel le Prophète explique à la fille d'Abou Bakr que la femme doit se couvrir, sauf son visage et ses mains, dont l'authenticité est cependant remise en cause. Enfin, il évoque le principe de précaution cher aux salafistes. Selon ce principe, si une action non interdite amène à une action interdite, la première action devient interdite. La non-dissimulation de la femme peut amener au péché et donc, pour éviter cela, il faut se couvrir. Il mentionne également le juriste Ibn Taymiyah selon lequel il faut dissimuler le visage. Enfin, il faut éviter que les musulmans ressemblent aux non-musulmans, et il invoque à ce propos un hadith disant que celui qui imite un groupe devient comme lui.

Cette courte épître d'Ibn Baz met en avant la méthode wahhabite qui revient directement au Coran et à la sunna. On utilise le principe de précaution, on interdit les imitations de non-musulmans et enfin, on cite Ibn Taymiyah $^{22}$.

\subsection{AL-QARADAWI : ABSENCE D'UN TEXTE AUTHENTIQUE ET UNIVOQUE}

Le cheikh al-Qaradawi est une autre grande figure du sunnisme contemporain, connu pour ses apparitions sur la chaîne de télévision Al-Jazeera et ses écrits prolifiques ${ }^{23}$. Dans une fatwa d'une vingtaine de pages prononcée par al-Qaradawi en octobre $1995^{24}$, des femmes musulmanes voilées s'adressent à lui en disant qu'il a défendu les femmes portant le niqab bien qu'il ne

21. Sur cette question, V. BURTON J., The sources of Islamic law. Islamic theories of abrogation, Edinburg University Press, 1990.

22. Le cheikh wahhabite Ibn Athaminyin (m. 2001) arrive au même résultat dans son épître (risala fi al-hijab). Dans une position à part, le cheikh syrien d'origine albanaise Al-Albani (m. 1999) revient sur les textes du Coran et des hadiths et conclut que la dissimilation du visage n'est pas obligatoire, tout en précisant bien qu'il ne s'agit pas de son opinion personnelle (Jilbab al-mara' al-muslima fi al-kitab wa al-sunna, Dar al-salam, s.d.).

23. Sur al-Qaradawi, V. notamment GräF B. and SkovgaArd-PAtersen J., Global mufti: the phenomenon of Yūsuf al-Qaraḍ̂awi, New-York, Columbia University Press, 2009.

24. http://www.feqhweb.com/vb/t5571.html [consulté le 3 juin 2016] 
le pense pas obligatoire, et elles lui demandent de défendre les muhajabat (femmes portant le hijab) et de déclarer qu'elles ne contredisent pas le Coran et la sunna. Ici on voit l'influence psychologique qu'exercent les tenants du voile intégral en mettant en doute l'islamité des femmes portant le hijab. Al-Qaradawi est conscient des divergences existant sur cette question et considère qu'elles perdurent même chez des musulmans croyants et sincères.

Il soutient tout d'abord que la non-obligation de couvrir le visage est l'opinion de la majorité des penseurs et cela depuis l'époque des compagnons du Prophète. Il rapporte notamment que les quatre écoles sunnites soutiennent l'opinion que le visage et les mains n'appartiennent pas au 'awra (parties intimes). Il rapporte les arguments de ceux qui interdisent la couverture du visage en se fondant sur l'interprétation du verset qui parle des atours apparents (zina zahira), certains penseurs suivant l'avis d'Ibn 'Abbas pour qui cela correspond au visage et aux mains et d'autres celui d'Ibn Mas'ud qui dit que cela correspond aux vêtements. Pour al-Qaradawi, à propos du verset 31 de la sourate La lumière, "rabattre leur khimar sur juyubihun », c'est-à-dire sur la poitrine, indique que le khimar concerne la couverture de la tête et que donc la couverture du visage n'est pas mentionnée. Un troisième argument repose là aussi sur le verset 30 de la sourate La lumière : si le visage était couvert, il n'y aurait pas cette injonction de ne pas regarder. Et à propos du verset 52 de la sourate Les coalisés qui mentionne les femmes qui peuvent plaire : comment vont-elles plaire si elles portent le niqab? Un autre argument s'appuie sur des exemples de femmes qui ne couvraient pas leurs visages au temps du Prophète. Il rapporte même un évènement où les compagnons du Prophète se sont étonnés qu'une femme porte le niqab. Enfin, si la femme veut vendre, acheter, être mandataire ou témoigner devant un tribunal, elle doit révéler son identité et, de fait, montrer son visage.

Pour al-Qaradawi, la thèse en faveur de l'obligation du niqab n'a pas de dalil sahih al-thubut et sarih al-dalala (une source certaine dans son existence et certaine dans son sens). Ici, il fait référence à la manière d'argumenter en droit musulman. Pour soutenir un argument certain, il faut que celui-ci soit basé sur un texte authentique et que son sens soit clair et univoque. Or selon lui, ceux qui défendent l'obligation de la dissimulation du visage ne peuvent s'appuyer sur aucun texte authentique et univoque.

Dans le verset 59 de la sourate Les factions, le mot «voile» (jilban) ne désigne pas forcément la couverture du visage. Dans le verset 31 de la sourate La lumière, « ce qui en paraît » a été traduit par les vêtements par Ibn Mas'ud, mais il y a une autre interprétation faite par Ibn 'Abbas qui renvoie 
au khôl et aux bagues. Ceux qui soutiennent l'obligation de couvrir le visage se basent sur fasd al 'asr (la corruption de l'époque) en affirmant que, même si les autres ont raison, on vit dans un monde corrompu au niveau moral et qu'il faut faire plus d'effort et interdire des choses qui auraient été permises dans un temps «normal». Cet argument de la corruption du temps (appelé aussi fasad al-zaman) est utilisé par les conservateurs pour durcir les règles du droit musulman, et affirmer que, si au temps du Prophète on permettait, par exemple, aux femmes de faire certaines choses, on ne le permet pas aujourd'hui en raison de la corruption des mœurs ${ }^{25}$.

Après avoir donné les arguments des deux camps, al-Qaradawi avance alors les siens propres en penchant nettement pour la solution de la nonobligation de la dissimulation du visage. Il dit pour l'essentiel que le principe est la liberté et que pour limiter cette liberté il faut qu'il y ait un texte clair et certain, ce qui n'est pas le cas ici. Si Dieu voulait faire de la dissimulation du visage une obligation, il l'aurait mentionné dans un texte univoque. Un autre argument majeur repose sur le principe du changement de la règle avec le changement d'époque (taghayyur al-ahkam fi taghayyur al-zaman), selon lequel il faut, selon lui, donner aujourd'hui plus de droits à la femme ${ }^{26}$.

\subsection{JAMAL AL-BANA : LE VOILE N'EST PAS OBLIGATOIRE}

Parmi les coranistes contemporains, nous mentionnerons Jamal al-Bana ${ }^{27}$, mort en 2013. Selon lui, il faut s'en tenir pour l'essentiel au Coran et les fuqaha' contemporains n'étant pas forcément plus intelligents que chacun d'entre nous, il ne faut pas donner plus de poids à leurs opinions qu'à nos propres opinions. Selon al-Bana, le voile est une coutume non musulmane que les musulmans ont adoptée. Il pense qu'il y a un islam «pur » qu'on peut séparer des éléments étrangers, de la même manière qu'opère un chimiste qui isole les matières : pour savoir si une règle appartient ou non à l'islam, il faut se référer au Coran. Ce qui est en accord avec le Coran, c'est l'islam et ce qui le contredit n'est pas l'islam. Le Coran est général et ne donne pas de détails. La sunna du Prophète est venue pour le compléter, mais pour ce

25. V. sur cette notion, Katz M. H., "The "corruption of the times" and the mutability of the Shari'a », Cardozo Law Review, vol. 28, 2006, p. 171-185.

26. Cette position de non-obligation de la dissimulation du visage est exprimée par une fatwa de la Maison égyptienne de la fatwa, fatwa n 374 de l'année 2012 : http://m.dar-alifta.org/ ViewFatwa.aspx? ID=4492\&LangID=1 [consulté le 3 juin 2016].

27. Sur son ouvre et sa vie, V. DuARTE S., L'idée de réforme religieuse en islam depuis les indépendances, thèse, EPHE, 2014, p. 120-133. 
qui ne correspond plus à son époque, on doit recourir au Coran. Puis, il rapporte la parole du Prophète selon laquelle «vous connaissez mieux que moi les affaires mondaines » et une parole d'un homme comme le Prophète ne peut être éternelle. Il considère de plus que des milliers de hadiths ont été falsifiés. Il ajoute que l'islam actuel est l'islam des fuqaha' et des écoles juridiques qui ont été établies il y a plus de mille ans. Ce qui a été fixé alors par les oulémas n'est plus valable aujourd'hui.

Après avoir énoncé ces principes, il analyse le texte coranique et arrive à la conclusion que le mot hijab dans le Coran ne signifie pas un vêtement, mais quelque chose qui sépare, comme une montagne ou une clôture du cœur, ou une séparation entre croyants et non-croyants le jour dernier, ou quelque chose derrière lequel on se cache. Plus spécifiquement, le verset 53 de la sourate Les factions se rapporte au fait que la maison du Prophète était liée à la mosquée et c'est pourquoi Dieu voulait que les musulmans ne rentrent pas dans la maison du Prophète sans autorisation. Le verset 31 de la sourate La lumière qui fait injonction aux musulmans de ne pas regarder les femmes confirme le fait que les femmes ne couvraient pas leur visage, sinon il n'y aurait aucune logique à énoncer ce commandement. Il présente donc les femmes au temps du Prophète comme ne portant pas le niqab et ne considère donc pas la dissimulation du visage comme obligatoire. Enfin, al-Bana accepte le hijab, mais pour lui il n'est pas obligatoire et la femme qui ne le porte pas ne se conduit pas dans un sens contraire à l'islam.

On voit donc s'exprimer des divergences de points de vue des juristes et penseurs musulmans sur la question de la dissimulation du visage et le passage d'une pensée juridique nuancée et casuistique à une pensée juridique aplatie et uniformisée, qu'elle soit libérale ou conservatrice. 